\title{
Effect of superlattice modulation of electronic parameters on the density of states of cuprate superconductors
}

\author{
Kai-Yu Yang, ${ }^{1}$ T. M. Rice, ${ }^{1,2}$ and Fu-Chun Zhang ${ }^{1}$ \\ ${ }^{1}$ Centre of Theoretical and Computational Physics and Department of Physics, The University of Hong Kong, Hong Kong \\ ${ }^{2}$ Institut für Theoretische Physik, ETH Zurich, CH-8093 Zürich, Switzerland
}

(Received 16 May 2007; revised manuscript received 11 August 2007; published 7 September 2007)

\begin{abstract}
Recent scanning tunneling microscopy on BSCCO 2212 has revealed a substantial spatial supermodulation of the energy gap in the superconducting state. We propose that this gap modulation is due to the superlattice modulations of the atoms in the structure, and hence the parameters in a microscopic model of the $\mathrm{CuO}_{2}$ plane. The gap modulation is estimated using renormalized mean field theory for a $t-t^{\prime}-J$ model on a superlattice. The results compare well with experiment.
\end{abstract}

DOI: 10.1103/PhysRevB.76.100501

PACS number(s): 74.72.Hs, 74.20.-z, 74.62.Bf, 74.81.-g

Scanning tunneling microscopy (STM) has become an important method to probe the electronic structure in high- $T_{c}$ cuprates. ${ }^{1-4}$ Recently STM has revealed a substantial modulation of the superconducting energy gap along the $a$ axis in BSCCO 2212 samples. ${ }^{5,6}$ The modulation appears in phase with the incommensurate periodic superlattice modulation which originates in the Bi-O layers. ${ }^{7,8}$ Structure studies show that this lattice modulation also affects the $\mathrm{CuO}_{2}$ planes, and so will modify the local electronic structure. Understanding this gap modulation may shed new light on our understanding of the superconductivity mechanism of high- $T_{c}$ cuprates. In this paper, we analyze the effect of modulating the parameters in a $t-t^{\prime}-J$ model on the superconducting state within the renormalized mean field theory (RMF). ${ }^{9}$ Our calculations show that the observed superconducting gap modulation can be explained by a reasonable choice for the parameter modulation within a $t-t^{\prime}-J$ model as a consequence of the short coherence length, although the precise form of a priori $t-t^{\prime}-J$ modulation will need more concrete structure data. Zhu ${ }^{10}$ has previously used a slave boson approximation to examine the effect of defect and acceptor induced parameter changes on superconductivity in the same model. Zhou et al. ${ }^{11}$ used RMF keeping $t, t^{\prime}$, and $J$ fixed spatially but adding local Coulomb potentials to model dopant oxygen acceptors close to the $\mathrm{CuO}_{2}$ planes to explain the nanoscale inhomogeneity found in STM experiments. In this paper we restrict our modeling to the superlattice structure. A somewhat different approach has been taken by Andersen, Hirschfeld, and Slezak $^{12}$ who introduce a periodic modulation of the pairing interaction in a conventional $d$-wave BCS Hamiltonian to describe the superlattice.

Shortly after the discovery of the BSCCO superconductor, an incommensurate lattice modulation with a period of $\approx 5$ unit cells along its $a$ axis was found and characterized. ${ }^{7,8}$ Note that this direction corresponds to a diagonal $(1,1)$ direction in the $\mathrm{CuO}_{2}$ square lattice. The determination of the detailed displacements and site occupation that occur in this superlattice modulation is difficult to undertake in a complex material such as BSCCO. While there is unanimity among the various superlattice structures reported in the literature $7,8,13,14$ that substantial modulations occur in the key $\mathrm{CuO}_{2}$ planes, there is no agreement about their precise forms. ${ }^{15}$ As a result, a priori estimates of the electronic pa- rameter modulation within a $t-t^{\prime}-J$ model are still not possible at present.

We start from a $t-t^{\prime}-J$ model on a square lattice including the no-double occupation constraint. Zhang and co-workers introduced the Gutzwiller approximation, replacing the nodouble occupation constraint by the classical statistical weight factors $g_{t}^{i, j}$ and $g_{s}^{i, j}$ for the hopping and spin-spin superexchange processes, respectively. ${ }^{9}$ The RMF that they derived reproduces the results of Variational Monte Carlo calculations on the full model quite well. Both have been proved to be capable of explaining many experiments qualitatively and in some cases even quantitatively. ${ }^{16}$ Decoupling the renormalized Hamiltonian gives a mean field form in the presence of pairing

$$
\begin{aligned}
H= & -\sum_{\langle i, j\rangle, \sigma} g_{t}^{i, j} t_{i, j} c_{i, \sigma}^{\dagger} c_{j, \sigma}-\sum_{\langle\langle i, j\rangle\rangle, \sigma} g_{t}^{i, j} t_{i, j}^{\prime} c_{i, \sigma}^{\dagger} c_{j, \sigma}-\mu \sum_{i, \sigma} c_{i, \sigma}^{\dagger} c_{i, \sigma} \\
& -\frac{3}{8} \sum_{\langle i, j\rangle, \sigma} g_{s}^{i, j} J_{i, j}\left[\chi_{i, j}^{*} c_{i, \sigma}^{\dagger} c_{j, \sigma}+\text { H.c. }\right] \\
& -\frac{3}{8} \sum_{\langle i, j\rangle, \sigma} g_{s}^{i, j} J_{i, j}\left[\Delta_{i, j}^{*}\left(c_{i, \uparrow} c_{j, \downarrow}-c_{i, \downarrow} c_{j, \uparrow}\right)+\text { H.c. }\right]
\end{aligned}
$$

where the operator $c_{i, \sigma}^{\dagger}\left(c_{i, \sigma}\right)$ creates (annihilates) an electron with spin $\sigma$ on the $i$ th lattice site. $t_{i, j}$ and $t_{i, j}^{\prime}$ are the hopping integrals for nearest neighboring (NN) sites $\langle i, j\rangle$ and nextnearest neighboring (NNN) sites $\langle\langle i, j\rangle\rangle$, respectively. $J_{i, j}$ is the NN spin-spin coupling constant, $\mu$ is the chemical potential, and $\chi_{i, j}=\sum_{\sigma}\left\langle c_{i, \sigma}^{\dagger} c_{j, \sigma}\right\rangle, \Delta_{i, j}=\left\langle c_{i, \uparrow} c_{j, \downarrow}-c_{i, \downarrow} c_{j, \uparrow}\right\rangle$ are the local NN particle-hole and particle-particle pairing fields. The Gutzwiller renormalization factors $g_{t}^{i, j}$ and $g_{s}^{i, j}$ depend on the local doping as ${ }^{17}$

$$
g_{t}^{i, j}=\sqrt{\frac{2 \delta_{i}}{1+\delta_{i}} \frac{2 \delta_{j}}{1+\delta_{j}}}, \quad g_{s}^{i, j}=\frac{4}{\left(1+\delta_{i}\right)\left(1+\delta_{j}\right)},
$$

where $\delta_{i}=1-n_{i}$ is the on-site hole concentration with $n_{i}$ $=\Sigma_{\sigma}\left\langle c_{i, \sigma}^{\dagger} c_{i, \sigma}\right\rangle$.

As discussed above, the incommensurate lattice modulation has a period of $\approx 5$ unit cells along the diagonal direction. In this paper we examine an electronic superlattice structure with a commensurate periodicity, i.e., each unit cell consists of 10 sites as illustrated in Fig. 1(a). This superlat- 

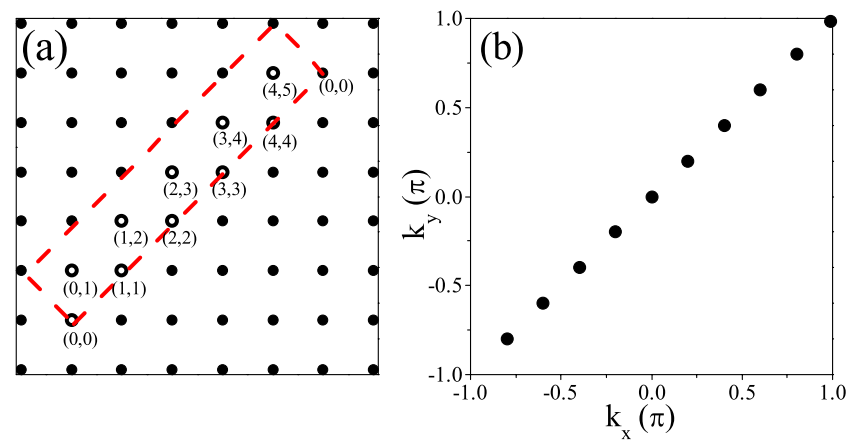

FIG. 1. (Color online) (a) The supercell structure. The black open dots present the sites in a unit cell with the coordinate inside the cell indicated. (b) The reciprocal lattice for a unit cell.

tice has a period of $5 \sqrt{2}$ lattice constants along the $(1,1)$ direction and $\sqrt{2}$ perpendicular to it. The reciprocal lattice is shown in Fig. 1(b). We use $\mathbf{I}(\mathbf{J})$ to index the supercell and $i(j)$ for the original lattice. The lattice constant is set as the length unit with $\boldsymbol{\tau}=(0, \pm 1)$ or $( \pm 1,0)$ for $\mathrm{NN}$, and $\boldsymbol{\Gamma}$ $=( \pm 1, \pm 1)$ for NNN. Here we assume a simple cosine modulation, consistent with the dominant lattice modulation observed in $\mathrm{Bi}-\mathrm{O}, \mathrm{Sr}$, and $\mathrm{CuO}_{2}$ layers,

$$
\begin{gathered}
t_{\boldsymbol{I}, \boldsymbol{I}+\boldsymbol{\tau}} d t_{0}=1+\frac{A}{2}\left\{\cos \left[\mathbf{Q} \cdot \mathbf{R}_{\boldsymbol{I}}+\cos \left(\mathbf{Q} \cdot \mathbf{R}_{\boldsymbol{I + \tau}}\right)\right]\right\}, \\
J_{\boldsymbol{I}, \boldsymbol{I}+\tau} / J_{0}=\left(t_{\boldsymbol{I}, \boldsymbol{I}+\boldsymbol{\tau}} / t_{0}\right)^{2}, \\
t_{\boldsymbol{I}, \boldsymbol{I}+\boldsymbol{\Gamma}}^{\prime} / t_{0}^{\prime}=1+\frac{B}{2}\left[\cos \left(\mathbf{Q} \cdot \mathbf{R}_{\boldsymbol{I + \Gamma}+\Gamma_{x}}\right)+\cos \left(\mathbf{Q} \cdot \mathbf{R}_{\boldsymbol{I}+\Gamma_{y}}\right)\right],
\end{gathered}
$$

where $\mathbf{Q}=(\pi / 5, \pi / 5)$ and $\mathbf{R}_{I}$ is the lattice coordinate within a unit cell. The average parameters are set to be $t_{0}$ $=300 \mathrm{meV}, J_{0} / t_{0}=0.3, t_{0}^{\prime} / t_{0}=-0.3$, comparable to the experimentally observed values. A quadratic relation between $t$ and $J$ is preserved unless specified otherwise. The Hamiltonian in Eq. (1) with the supercell structure is solved selfconsistently. The local density of states (LDOS) $N_{I}(\omega)$ is determined by a Fourier transformation of the local Green's function $i G_{I, \sigma}=\left\langle T_{\tau}\left[c_{I, \sigma}^{\dagger}(\tau) c_{I, \sigma}(0)\right]\right\rangle$ multiplied by a renormalization factor $g_{t}^{\mathbf{I}, \mathbf{I}}$, where $T_{\tau}$ is the time ordering operator.

Our simple model with modulations of $t, t^{\prime}$, and $J$ can reproduce many of the most prominent features of the STM experiments. The variation of the gap (about 10\%) within a wide doping range is reproduced by a reasonable choice of the electronic parameter modulation in our theory. It is worth noting that our model gives a very weak doping dependence of the relative gap variance, which agrees well with the data recently reported by the Cornell group. ${ }^{5,6}$ In addition, the low-energy subgap spectra are spatially homogeneous despite the inhomogeneity in the gap. Further, the coherent peaks are always almost symmetrically located around zero bias. The gap is negatively correlated with the local doping concentration. In our calculation, we have normalized the gap value obtained from the RMF by a factor of 2 to bring them in line with the more accurate Variational Monte Carlo results. ${ }^{16}$
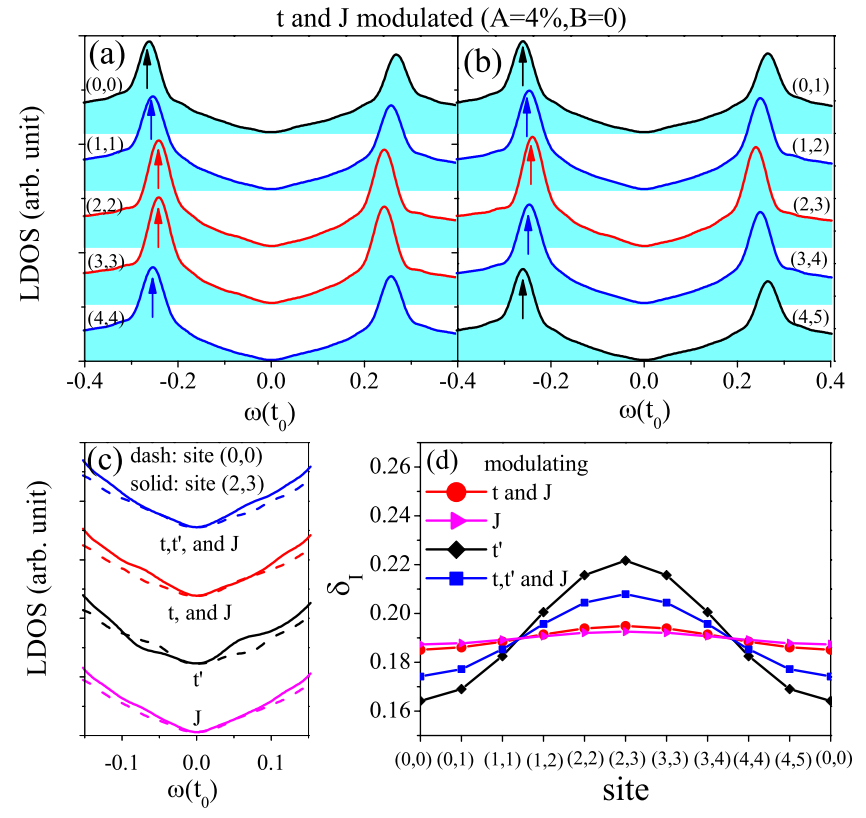

FIG. 2. (Color online) Panels (a), (b) On-site LDOS with $t$ and $J$ modulated $[A=4 \%, B=0$ in Eq. (3) $]$ with the peak positions indicated by arrows. The energy $\omega$ is in units of $t_{0}(=300 \mathrm{meV})$. Panel (c) The LDOS at low energies at two supercell sites $(0,0)$ and $(2,3)$, and panel (d) for on-site local hole concentration. The doping is $\delta=0.19$. In (c), (d), $A=4 \%$ in the modulations of $t$ and $J$ modulation (red), and $J$ only with $t$ fixed (magenta), $B=45 \%$ in the modulation of $t^{\prime}$ (black), and $A=2 \%$ and $B=22.5 \%$ in the modulation of $t, t^{\prime}$, and $J$ (blue).

To compare with the recent STM experiments, three typical doping levels with $\delta=0.13$ (UD), 0.16 (OP), 0.19 (OD) are considered. In our numerical calculation, a $1000 \times 400$ supercell structure is introduced. The LDOS presented here is filtered by fast Fourier transformation with an energy window of $11 \mathrm{meV}$. We have studied the effects of various possible modulations of parameters $t, t^{\prime}$, and $J$ to the LDOS and to the local gap values. The effects are qualitatively similar, although it is much less sensitive to vary $t^{\prime}$ than $t$ or $J$.

First we consider modulation of $t$ and $J$ while keeping $t^{\prime}$ unchanged, where the modulation amplitudes $A=4 \%$ and $B=0$. The resulting variances of $t$ and $J$ are $\sim 7$ and $\sim 14 \%$, respectively. The results shown in Fig. 2 are for doping 0.19, which are representative for all the three hole concentrations studied here. The LDOS is shown in Figs. 2(a) and 2(b). At low energy there is a clear homogeneous "V" shape [panel(c)] indicating the nodal structure $\left(v_{F}, v_{\Delta}\right)$ is robust against this electronic modulation. The homogeneity in LDOS at low energy was also found by Wang et al., ${ }^{17}$ in studies of disorder effects. The coherent peaks with lower height are located at higher energy, and the spectral weight suppressed at low energy is transferred to high energy near the band edge. Note that there is a multipeak character of the LDOS which is smeared by fast Fourier transformation. Similar multipeak character has also been observed in a recent STM experiment with high-energy resolution up to $2 \mathrm{meV} .{ }^{18}$ The modulation of the superconducting energy gap [shown in Fig. 3(a1)] has a variation about $10 \%$ for this parameter choice, which matches well with recent STM experiments. The doping 


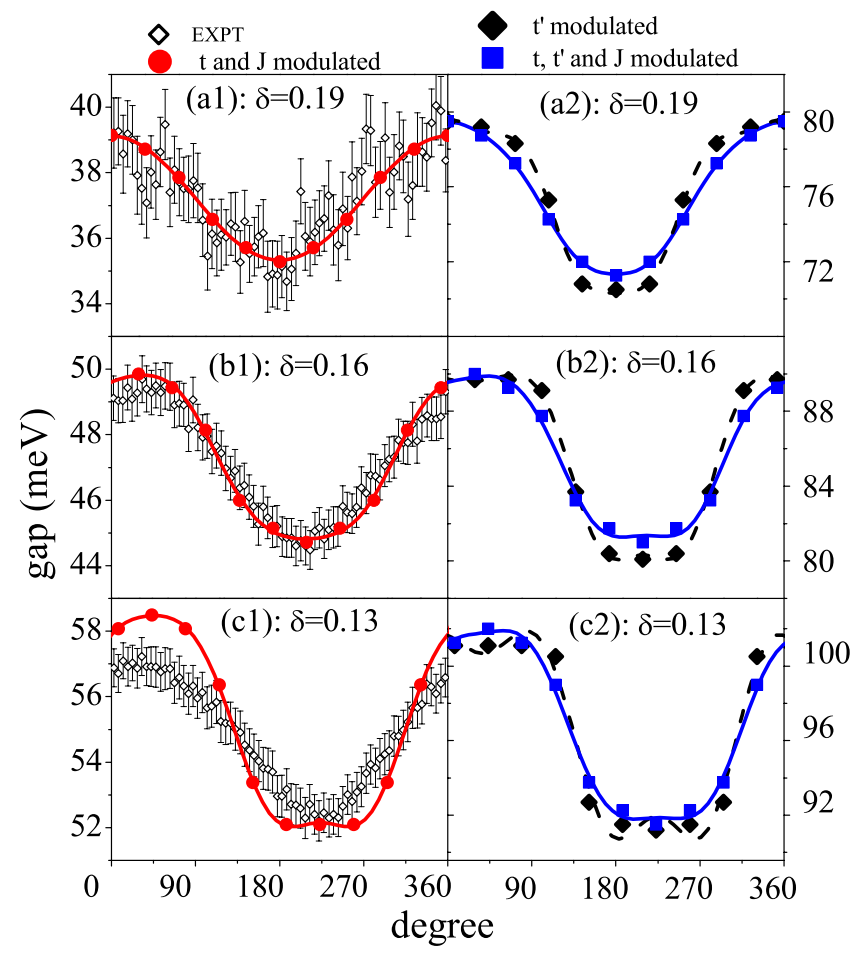

FIG. 3. (Color online) The gap modulation due to electronic parameter modulations. The phase $\phi$ characterizes the electronic structure supermodulation which can be described by a cosine form. Experimentally the supermodulation phase mapping $\phi(r)$ is consistent with the original topograph $Z(r)$. (Refs. 5 and 6). The theoretical gap modulation vs $\mathbf{Q} \cdot \mathbf{R}_{I}$ (the range is linearly scaled to $360^{\circ}$ ) is shifted along the horizonal direction to match the gap minimum and maximum loci. The open diamonds with error bars in panels (a1)(c1) represent STM experimental data (Refs. 5 and 6) for three samples (OD,OP,UD) with the energy gap $\Delta=37,47,55 \mathrm{meV}$, respectively. Solid symbols are from our calculations, red circles for $t$ and $J$ modulation $(A=4 \%, B=0)$, black diamonds for $t^{\prime}$ modulation ( $A=0, B=45 \%$ ), and blue squares for $t, t^{\prime}$, and $J$ modulations $(A=2 \%, B=22.5 \%)$. Color curves show analytic interpolations of our calculations between lattice sites.

variance achieved here is about 5\%, i.e., $0.185-0.195$ [shown in Fig. 2(d)], comparable with the value of $7 \%$ recently reported in the STM experiments., ${ }^{419}$ The negative correlation between the gap and the doping concentration is reproduced. In addition, there is a small deviation from the original $d$-wave symmetry due to the symmetry breaking imposed by the supercell structure orientation rotated by $45^{\circ}$. In Figs. 3(a1)-3(c1) the modulations of the gap for different dopings are shown compared with the STM results. The phase $\phi$ serving as the horizonal axis characterizes the electronic structure supermodulation, which can be described by a consine form. The supermodulation phase mapping $\phi(r)$ is consistent with the observed original topograph $Z(r) .5,6$ The theoretical gap modulation vs $\mathbf{Q} \cdot \mathbf{R}_{I}$ (the range is linearly scaled to $360^{\circ}$ ) is shifted along the horizonal direction to match the gap minimum and maximum loci. Keeping the $t$ and $J$ modulation constant, a very weak doping dependence of the variance of the gap modulation is observed. Both the form and the magnitude of the gap modulation agree well with the experimental results on samples ranging between the underdoped and overdoped regime. The decreased agreement happened at low doping $(\delta=0.13)$ is mainly due to the larger peak-to-peak distance of the on-site LDOS, which possesses a multipeak structure before fast Fourier transformation.

We have also considered the superlattice modulations of $t^{\prime}$ only, $J$ only, and a combined modulation of $t-t^{\prime}-J$. The low-energy LDOS and the local hole density for the various choices of the modulated parameters are shown in Figs. 2(c) and 2(d), respectively. In Figs. 3(a)-3(c), we show the gap modulation obtained from the various choices of parameter modulations. It requires a substantial modulation of $t^{\prime}$, i.e., $A=0$ and $B=45 \%$, corresponding to a range for $t^{\prime}=0.165-0.435$, to have similar gap modulation with $\sim 10 \%$ change in gap. The large variation in local hole density is due to the large modulation of $t^{\prime}$ required to obtain this modulation of the gap. By modulating $J(A=4 \%)$ alone with the quadratic relation between $t$ and $J$ released, we have found only a slight difference from the case with both $t$ and $J$ modulated simultaneously. Hence the results for modulating $J$ alone are omitted in Fig. 3 due to the close similarity to modulating $t$ and $J$ simultaneously. The low-energy behavior becomes more homogeneous and the variation of the doping concentration shrinks as shown in Figs. 2(c) and 2(d). Zhu, who examined the nanoscale inhomogeneity, ${ }^{10}$ presented a similar picture arguing that the presence of the randomly distributed out-of-plane dopant oxygens will lead to a change in the superexchange strength. From the cases studied above, we can reach a conclusion that, with similar variance of the superconducting energy gap, a modulation of $t^{\prime}$ leads to a larger modulation of doping concentration while $J$ and/or $t$ tend to give a better low-energy homogeneity. We have examined a combined modulation for a choice of $A=2 \%$ and $B=22.5 \%$. The LDOS, superconducting energy gap, doping concentration, all show an additive behavior from the individual modulation of $A$ and $B$ with little interference, and the low-energy LDOS becomes more homogeneous. From our current study it is obvious that modulation of the electronic parameters $t, t^{\prime}$, and $J$ is able to generate the superlattice modulation phenomenon recently observed in STM. 5,6

Pavarini et al. ${ }^{20}$ have proposed that the variation of the transition temperature $T_{c}$ between different cuprate families is controlled by the $\mathrm{Cu}-\mathrm{O} 3$ apical distance through its effect on the NNN hopping parameter $t^{\prime}$. The energy gap estimated by the RMF theory shows a similar dependence on $t^{\prime}$, namely, the gap increases with increasing values of $\left|t^{\prime}\right|$ at constant hole doping in agreement with the increase in $T_{c}$ argued by Pavarini et $a .^{20}$ It is therefore appealing to argue that this is the controlling feature of the superlattice modulation. However, there is a real problem with this interpretation. Slezak in his thesis ${ }^{5}$ finds that if he uses the $\mathrm{Cu}-\mathrm{O} 3$ distance modulation reported by Yamamoto et al. ${ }^{14}$ there is a negative correlation, i.e., smaller values of the gap occur at larger $\mathrm{Cu}-\mathrm{O} 3$ distances. This is opposite to the conclusion of Pavarini et al. ${ }^{20}$ that $\left|t^{\prime}\right|$ scales with the $\mathrm{Cu}-\mathrm{O} 3$ distance. Thus the simplest interpretation that modulation of $\left|t^{\prime}\right|$ is the controlling factor cannot be justified. Instead one cannot directly relate the reported structural data and the gap modulation. One can say, however, that the size of the modulations in the electronic parameters needed to explain the energy gap 
modulations are quite reasonable even if we cannot identify the specific values of individual parameter modulations.

Lastly we comment on a couple of related issues. Slezak in his thesis ${ }^{5}$ also reports on modulations of the bosonic mode $\Omega$ accompanying the superlattice modulations. However, as he points out, the isotope shift of $\Omega$ has no effect on the gap, which leads to the conclusion that both vary as a consequence of the structural modulation as was also suggested by Pilgram et al. ${ }^{21}$ A second feature of the BSCCO cuprates is the substantial disorder in the STM gap maps ${ }^{4}$ This is characterized by regions with enhanced gap values associated with a high-energy resonance in the STM spectra which has been interpreted as originating in a nearby dopant $\mathrm{O}^{2-}$ ion. ${ }^{4}$ This leads to a problem since an enhanced hole density would be expected around a $\mathrm{O}^{2-}$ ion, but the enhanced gap suggests a locally reduced hole density. Several proposals have been made to reconcile theses two features. ${ }^{10,11,19}$ However, Yamamoto et $a l .{ }^{14}$ and also Eisaki et $a .^{22}$ conclude that in BSCCO a substantial number of $\mathrm{Bi}$ ions substitute for the $\mathrm{Sr}$ ions on planes adjacent to the $\mathrm{CuO}_{2}$ planes. This suggests to us as it did to Eisaki et al., ${ }^{22}$ that since $\mathrm{Bi}^{3+}$ acts as a donor ion, the regions of enhanced gap should be associated with $\mathrm{Bi}^{3+}$ donors rather than $\mathrm{O}^{2-}$ acceptors.

We are very grateful to Séamus Davis and S. Uchida for stimulating discussion. This work was supported by the RGC grant and Centre of Theoretical and Computational Physics of HKSAR, RGC Central Allocation Grant of HKSAR, The University of Hong Kong, and the MANEP program of the Swiss National Foundation.
${ }^{1}$ S. H. Pan, J. P. O’Neal, R. L. Badzey, C. Chamon, H. Ding, J. R. Engelbrecht, Z. Wang, H. Eisaki, S. Uchida, A. K. Gupta, K.-W. $\mathrm{Ng}$, E. W. Hudson, K. M. Lang, and J. C. Davis, Nature (London) 413, 282 (2001).

${ }^{2}$ K. M. Lang, V. Madhavan, J. E. Hoffman, E. W. Hudson, H. Eisaki, S. Uchida, and J. C. Davis, Nature (London) 415, 412 (2002)

${ }^{3}$ Michael Vershinin, Shashank Misra, S. Ono, Y. Abe, Yoichi Ando, and Ali Yazdani, Science 303, 1995 (2004).

${ }^{4}$ K. McElroy, Jinho Lee, J. A. Slezak, D.-H. Lee, H. Eisaki, S. Uchida, and J. C. Davis, Science 309, 1048 (2005).

${ }^{5}$ J. C. Davis, URL http://online.kitp.ucsb.edu/ online/ coldatoms07/davis1/; J. Slezak, Ph.D. thesis, Cornell University, available at http://people.ccmr.cornell.edu/ jcdavis/theses/ james/

${ }^{6}$ J. A. Slezak et al. (unpublished).

${ }^{7}$ Yan Gao, Peter Lee, Philip Coppens, M. A. Subramanian, and A. W. Sleight, Science 241, 954 (1988).

${ }^{8}$ E. A. Hewat, M. Dupuy, P. Bordet, J. J. Capponi, C. Chaillout, J. L. Hodeau, and M. Marezio, Nature (London) 333, 53 (1988).

${ }^{9}$ F. C. Zhang, C. Gros, T. M. Rice, and H. Shiba, Semicond. Sci. Technol. 1, 36 (1988).

${ }^{10}$ Jian-Xin Zhu, arXiv:cond-mat/0508646 (unpublished).

${ }^{11}$ Sen Zhou, Hong Ding, and Ziqiang Wang, Phys. Rev. Lett. 98, 076401 (2007).

${ }^{12}$ Brian M. Andersen, P. J. Hirschfeld, and James A. Slezak, Phys. Rev. B 76, 020507 (2007).

${ }^{13}$ D. Grebille, H. Leligny, A. Ruyter, Ph. Labbé, and B. Raveau,
Acta Crystallogr., Sect. B: Struct. Sci. 52, 628 (1996).

${ }^{14}$ Akiji Yamamoto, Mitsuko Onoda, Eiji Takayama-Muromachi, Fujio Izumi, Toru Ishigaki, and Hajime Asano, Phys. Rev. B 42, 4228 (1990).

${ }^{15}$ For example, Grebille et al. (Ref. 13) report a large vacancy wave on the $\mathrm{O} 3$ apical oxygen site. Yamamoto et al. (Ref. 14) find full occupancy on this site but substantial numbers of $\mathrm{Bi}$ and $\mathrm{Ca}$ ions on the $\mathrm{Sr}$ sites which are in the same plane.

${ }^{16}$ P. W. Anderson, P. A. Lee, M. Randeria, T. M. Rice, N. Trivedi, and F. C. Zhan, J. Phys.: Condens. Matter 16, R755 (2004); Kai-Yu Yang, C. T. Shih, C. P. Chou, S. M. Huang, T. K. Lee, T. Xiang, and F. C. Zhang, Phys. Rev. B 73, 224513 (2006); Arun Paramekanti, Mohit Randeria, and Nandini Trivedi, ibid. 70, 054504 (2004).

${ }^{17}$ Qiang-Hua Wang, Z. D. Wang, Yan Chen, and F. C. Zhang, Phys. Rev. B 73, 092507 (2006).

${ }^{18}$ A. C. Fang, L. Capriotti, D. J. Scalapino, S. A. Kivelson, N. Kaneko, M. Greven, and A. Kapitulnik, Phys. Rev. Lett. 96, 017007 (2006).

${ }^{19}$ Tamara S. Nunner, Brian M. Andersen, Ashot Melikyan, and P. J. Hirschfeld, Phys. Rev. Lett. 95, 177003 (2005).

${ }^{20}$ E. Pavarini, I. Dasgupta, T. Saha-Dasgupta, O. Jepsen, and O. K. Andersen, Phys. Rev. Lett. 87, 047003 (2001).

${ }^{21}$ S. Pilgram, T. M. Rice, and M. Sigrist, Phys. Rev. Lett. 97, 117003 (2006).

${ }^{22}$ H. Eisaki, N. Kaneko, D. L. Feng, A. Damascelli, P. K. Mang, K. M. Shen, Z.-X. Shen, and M. Greven, Phys. Rev. B 69, 064512 (2004). 